\title{
Aberrant Internal Carotid Artery: Clinical Implications
}

\section{Vipin Kumar Garsa ${ }^{1}$, Nidhi Sheokand 2, Sunil Saharan *3, Vivek Singh Malik ${ }^{1}$.}

${ }^{1}$ Associate Professor, Department of Anatomy, Pt. B.D.S. PGIM SUniversity, Rohtak, Haryana, India.

2 Resident, Department of Anesthesiology, New York M ethodist Hospital, Brooklyn, NY 11215.

${ }^{*}$ Assistant Professor, Division of Pediatric Cardiology, Department of Pediatrics, New York University, New York, NY, 10016.

\section{ABSTRACT}

Abstract: Anatomical variations in the course of internal carotid artery are of paramount clinical importance to the physicians in the field of anesthesia and oral surgery. We describe here an interesting case of sinuous loop of right internal carotid artery discovered during dissection study. The dissection study provided an excellent opportunity for analyses of anatomical relations and morphometry, which is usually not possible with cross sectional imaging. The upper portion of the loop was extending into the retropharyngeal space at the level of soft palate. Anatomical relationship and histology of aberrant internal carotid artery were studied in detail. Clinical implications of aberrant internal carotid artery especially for the clinicians are discussed in detail.

KEY WORDS: Retropharyngeal, Aberrant, Internal Carotid Artery, Complication, Clinical Implication.

Address for correspondence, Sunil Saharan, MD, Assistant Professor, Division of Pediatric Cardiology, Department of Pediatrics, New York University, 403 E 34th St, New York NY 10016 E-Mail: sunil.saharan@ nyumc.org

\begin{tabular}{|c|c|}
\hline \multicolumn{2}{|r|}{ Online Access and Article Informtaion } \\
\hline \multirow{2}{*}{ Quick Response code } & $\begin{array}{c}\text { International Journal of Integrative Medical Sciences } \\
\text { www.imedsciences.com }\end{array}$ \\
\hline & 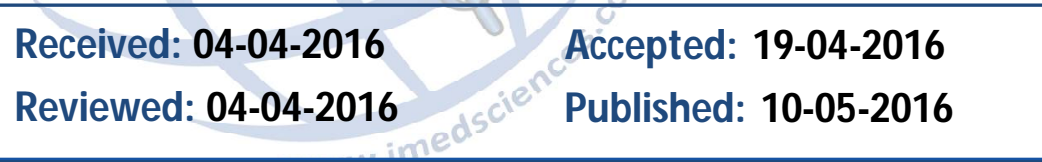 \\
\hline Source of Funding: Self & Conflicts of interest: None \\
\hline
\end{tabular}

\section{BACKGROUND}

Internal carotid arteries are a major source of blood supply to the brain along with contributions from vertebral arteries. Usually each internal carotid artery arises from bifurcation of common carotid artery at the level of hyoid bone. The segment of internal carotid artery from the bifurcation to the skull base is called the cervical segment which is further divided into a lower or sternomastoid portion and an upper or parapharyngeal portion, and in most individuals it is lateral to the pharyngeal wall. These arteries develop from the dorsal aorta and third aortic arch. Initially, these arches are located in the region of primitive pharynx and later during eighth week of embryonic period dorsal aorta descends to the thorax. Due to thoracic descent, course of the carotid artery transforms from an arch into a straight vessel. Partial failure of thoracic descent is responsible for occurrence of kinks and loops in carotid artery. M edial displacement of the artery due to kinks and loops can bring it into retropharyngeal or even midline location. Cases have been reported in which bifurcation took place at the levels of the upper or middle portions of the thyroid cartilage and at the level of the cricoid cartilage. In extremely rare case, external and internal carotids may arise independently from the subclavian artery and therefore there may be no common carotid artery [1].

Incidence of non-straight carotid arteries has been estimated to be $2 \%$ [2]. Most cases of aberrant carotid arteries that have been reported 
were either found as a surprise during surgeries involving neck region or during imaging [3-6]. In a prior study on adult cadavers with 282 specimens, different variations in shape of internal carotid artery were: no curvature in $67.7 \% ; 26.2 \%$ had medial, lateral, or ventrocaudal curve; $4.2 \%$ showed kinking and $1.7 \%$ had coiling [7].

In such cases detailed three-dimensional analyses of anatomical relations, morphometry as well as documentation is deficient due to the demands of procedure or limitations of imaging technology. Thus finding of such a case in cadaveric dissection provided excellent opportunity for necessary measurements and reporting. This variation is of clinical significance for practitioners of anesthesia and oral surgery.

\section{CASE REPORT}

Right internal carotid artery in this case had a sigmoid tortuosity. Convexity of the sigmoid loop was facing laterally in its lower part and medially in its upper part (Fig. 1). This medially directed convexity extended between pharynx and pre-vertebral muscles at the level of soft palate. At this place internal carotid artery came in direct contact with the superior constrictor muscle. The relations of internal carotid artery were noted in this case. Near the base of the skull 9, 10 and 11th cranial nerves were posterior to the right internal carotid artery in that order. Glossopharyngeal nerve crossed the internal carotid artery laterally from posterior to anterior aspect. This nerve crossed the posterior auricular artery medially to the pharynx. Stylopharyngeus muscle followed the same course as of the glossopharyngeal nerve.

Posterior auricular artery crossed the internal carotid artery laterally from anterior to posterior direction at the crossing of glossopharyngeal nerve. Near the opening of jugular foramen vagus nerve was behind the right internal carotid artery and pharyngeal branch of vagus nerve crossed right internal carotid artery laterally from posterior to anterior direction to reach pharynx. Crossing of pharyngeal branch of vagus was inferior to crossing of glossopharyngeal nerve. Inferior to this level hypoglossal nerve passed in a lateral relation to right internal carotid artery. At this place hypoglossal nerve gave off superior root of Ansa cervicalis, which was in lateral relation to internal carotid artery up to its origin. Superior laryngeal branch of vagus nerve crossed internal carotid artery medially from posterior to anterior direction. Occipital artery crossed internal carotid artery laterally from anterior to posterior direction, with hypoglossal nerve sandwiched between these two structures. Posterior belly of digastric, stylohyoid muscle, and stylohyoid ligament were lateral to the internal carotid artery. Internal jugular vein was lateral to the artery in its lower part and posterior to artery near the jugular foramen.

Fig. 1: Sigmo id shaped internal carotid artery along with common \& external carotid arteries.

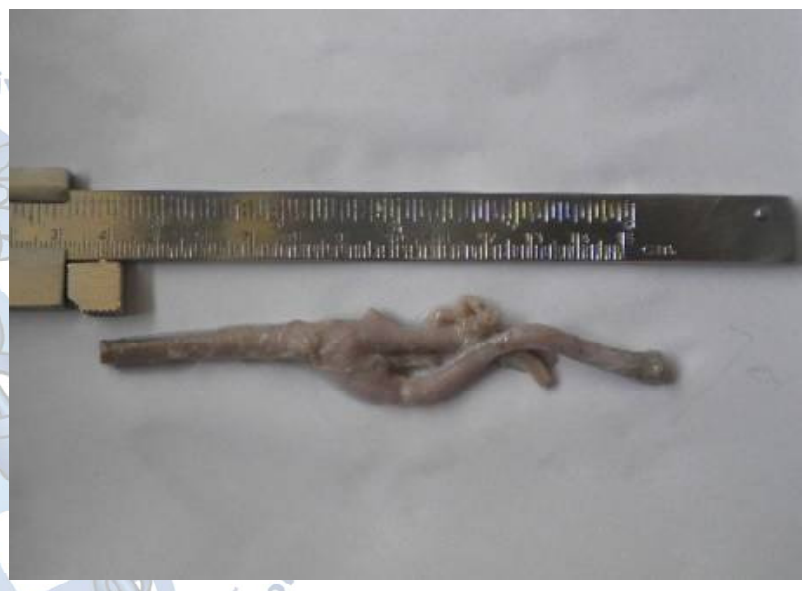

\section{DISCUSSION}

Complications associated with retropharyngeal internal carotid artery have been well documented in literature in past. There are studies with reported lacerations of posterior wall of pharynx extending to sub-mucosal soft tissue during nasal and oral endotracheal intubations leading to fatal hemorrhage $[8,9]$. Retropharyngeal internal carotid artery is also vulnerable to injury during adeno-tonsillectomy, uvulopalatopharyngoplasty and other pharyngeal surgeries [10]. Other clinical scenarios which can result in similar injury include transesophageal echocardiogram probe insertion and placement of nasogastric or orogastric tube $[11,12]$. Patients with retropharyngeal internal carotid artery are also predisposed to vascular insufficiency especially with change in position, cricoid pressure and obstructive sleep apnea [13]. During transoral approach to block the glossopharyngeal nerve in the pharynx, accidental arterial injection of 
local anesthetic or puncture of internal carotid may lead to serious complications. As aberrant internal carotid arteries are significantly associated with velocardiofacial syndrome, it is proposed that these patients should be routinely evaluated by magnetic resonance angiography preoperatively [14]. Loops or kinks associated with microscopic pathological findings are usually managed either by excision and anastomosis of remaining segments, total replacement of the looped arterial segment with venous homograft or by total mobilization of the loop along with resection of redundant proximal segment and reimplantation onto the bifurcation of carotid artery.

\section{CONCLUSION}

To conclude, one should always ask for history of difficulty swallowing, fullness of throat and known history of velocardiofacial syndrome before any pharyngeal procedures. Detailed physical examination should be done specifically evaluating for facial dysmorphism, palatal swelling or asymmetry, visible pharyngeal pulsations and ensuring bilateral carotid pulses on palpation in order to avoid catastrophic complication.

\section{REFERENCES}

[1]. Boyd JD. Absence of the Right Common Carotid Artery. J. Anat. 1934 Jul;68:551-557.

[2]. M arcucci C, Thomas P, Sewell DA. Retropharyngeal carotid artery: an important anatomic variation for the anesthesiologist. Anesthesiology. 2009 Aug;111:454-455.

[3]. Chen SS, Shao KN, ChiangJH, Chang CY, Lao CB, Lirng JF, Teng MM. Aberrant cervical carotid artery. Zhonghua Yi Xue Za Zhi (Taipei). 2000 Aug;63:653657.

[4]. Hertzanu Y, Tovi F. Radiology case of the month. Aberrant internal carotid artery manifesting as a pharyngeal mass. J Otolaryngol. 1992 Aug;21:294296.
[5]. Miller S, Weill A, Maillard JC, Miaux Y, Chiras J. Aberrant internal carotid artery presenting in the midline retropharyngeal space: case report. Can Assoc Radiol J. 1997 Feb;48:33-37.

[6]. Shankar L, Mehta AL, Hawke M, Rutka J. Highresolution computed tomography of an aberrant internal carotid artery. J Otolaryngol. 1992 Oct;21:373-375.

[7]. Paulsen F, Tillmann B, Christofides C, Richter W, Koebke J. Curving and looping of the internal carotid artery in relation to the pharynx: frequency, embryology and clinical implications. J. Anat. 2000 Oct;197 Pt 3:373-381.

[8]. Postma GN, Buenting JE, Jones KR. Oropharyngeal perforation after traumatic intubation. Otolaryngol Head Neck Surg. 1995 Sep;113:290-292.

[9]. Landess WW. Retropharyngeal dissection: a rare complication of nasotracheal intubation revisited-a case report. AANA J. 1994 Jun;62:273277.

[10]. Palacios E, Kirsch D, Rojas R. Anomalous course of the carotid arteries in the retropharyngeal space poses a surgical risk. Ear Nose Throat J. 2005 Jun;84:336-337.

[11]. Spahn DR, Schmid S, Carrel T, Pasch T, Schmid ER. Hypopharynx perforation by a transesophageal echocardiography probe. Anesthesiology. 1995 Feb;82:581-583.

[12]. M axeiner H. [Pressure damage of the hypopharynx caused by stomach tubes]. Beitr. Gerichtl. Med. 1990;48:123-133.

[13]. Tsuiki S, Isono S, Ishikawa T, Yamashiro Y, Tatsumi $\mathrm{K}$, Nishino T. Anatomical balance of the upper airway and obstructive sleep apnea. Anesthesiology. 2008 Jun;108:1009-1015.

[14]. Oppenheimer AG, Fulmer S, Shifteh K, Chang J-K, Brook A, Shanske AL, Shprintzen RJ. Cervical vascular and upper airway asymmetry in Velocardio-facial syndrome: correlation of nasopharyngoscopy with MRA. Int. J. Pediatr. Otorhinolaryngol. 2010 Jun;74:619-625.

How to cite this article:

Vipin Kumar Garsa, Nidhi Sheokand, Sunil Saharan, Vivek Singh Malik. Aberrant Internal Carotid Artery: Clinical Implications. Int J Intg Med Sci 2016;3(4):270-272. DOI: 10.16965/ijims.2016.114 